\title{
Common Fixed Point Results for Occasionally Weakly Compatible Maps in G-Symmetric Spaces
}

\author{
Kanayo Stella Eke ${ }^{1}$, Johnson Olaleru ${ }^{2}$ \\ ${ }^{1}$ Department of Computer and Information Science/Mathematics, Covenant University, Ota, Nigeria \\ ${ }^{2}$ Department of Mathematics, University of Lagos, Lagos, Nigeria \\ Email: ugbohstella@yahoo.com, olaleru1@yahoo.co.uk
}

Received 4 October 2013; revised 4 November 2013; accepted 14 November 2013

Copyright $(2014$ by authors and Scientific Research Publishing Inc.

This work is licensed under the Creative Commons Attribution International License (CC BY).

http://creativecommons.org/licenses/by/4.0/

(c) (i) Open Access

\section{Abstract}

The notion of a G-symmetric space is introduced and the common fixed points for some pairs of occasionally weakly compatible maps satisfying some contractive conditions in a G-symmetric space are proved. The results extend and improve some results in literature.

\section{Keywords}

Common Fixed Points; Generalized Contractive Mappings; Occasionally Weakly Compatible Maps; Compatible Maps; (E-A) Property; G-Symmetric Spaces

\section{Introduction}

The notion of metric spaces is widely used in fixed point theory and applications. Different authors had generalized the notions of metric spaces. Recently, Eke and Olaleru [1] introduced the concept of G-partial metric spaces by introducing the non-zero self-distance to the notion of G-metric spaces. The G-partial metrics are useful in modeling partially defined information which often appears in Computer Science. The concept of symmetric spaces in which the triangle inequality of a metric space is not included was introduced by Cartan [2] and defined as:

A symmetric on a set $X$ is a real valued function $d$ on $X \times X$ such that

(i) $d(x, y) \geq 0$ and $d(x, y)=0$ if and only if $x=y$;

(ii) $d(x, y)=d(y, x)$.

Wilson [3] also gave two more axioms of a symmetric $d$ on $X$ as:

$\left(W_{1}\right)$ Given $\left\{x_{n}\right\}, x$ and $y$ in $X, d\left(x_{n}, x\right) \rightarrow 0$ and $d\left(x_{n}, y\right) \rightarrow 0$ imply that $x=y$; 
$\left(W_{2}\right)$ Given $\left\{x_{n}\right\},\left\{y_{n}\right\}$ and $x \in X ; d\left(x_{n}, x\right) \rightarrow 0$ and $d\left(x_{n}, y_{n}\right) \rightarrow 0$ imply that $d\left(x_{n}, y\right) \rightarrow 0$.

Hicks and Rhoades [4] observed that the use of the triangle inequality is not necessary in certain proof of metric theorems. Based on this idea, they proved some common fixed point results in symmetric spaces.

Different generalizations of the metric space have been introduced by many authors in literature. In particular, Mustafa and Sims [5] generalized the concept of a metric space by assigning a real number to every triplet of an arbitrary set. Thus, it is defined as:

Definition 1.1 [5]: Let $X$ be a nonempty set, and let $G: X \times X \times X \rightarrow R^{+}$be a function satisfying:

$\left(G_{1}\right) G(x, y, z)=0$ if $x=y=z$,

$\left(G_{2}\right) \quad 0<G(x, x, y)$ for all $x, y \in X$ with $x \neq y$,

$\left(G_{3}\right) G(x, x, y) \leq G(x, y, z)$ for all $x, y, z \in X$ with $y \neq z$,

$\left(G_{4}\right) G(x, y, z)=G(x, z, y)=G(y, z, x)$ (symmetry in all three variables),

$\left(G_{5}\right) G(x, y, z) \leq G(x, a, a)+G(a, y, z)$ for all $x, y, z, a \in X$ (rectangle inequality).

Then, the function $G$ is called a generalized metric, or more specifically a G-metric on $X$, and the pair $(X, G)$ is a $\mathrm{G}$-metric space.

Example 1.2 [5]: Let $(X, d)$ be a metric space. The function $G: X \times X \times X \rightarrow[0, \infty)$, defined by

$$
G(x, y, z)=\max \{d(x, y), d(y, z), d(z, x)\}
$$

or

$$
G(x, y, z)=d(x, y)+d(y, z)+d(z, x)
$$

for all $x, y, z \in X$, is a G-metric on $X$.

In this work, we generalize the symmetric spaces by omitting the rectangle inequality axiom of G-metric space. This leads to our introduction of the notion of a G-symmetric space defined as follows:

Definition 1.3: A G-symmetric on a set $X$ is a function $G_{d}: X \times X \times X \rightarrow R^{+}$such that for all $x, y, z \in X$, the following conditions are satisfied:

$G_{d}(1) G_{d}(x, y, z) \geq 0$ and $G_{d}(x, y, z)=0$, if $x=y=z$;

$G_{d}(2) 0<G_{d}(x, x, y)$ for all $x, y \in X$ with $x \neq y$;

$G_{d}(3) G_{d}(x, x, y) \leq G_{d}(x, y, z)$, for all $x, y, z \in X$ with $y \neq z$;

$G_{d}(4) G_{d}(x, y, z)=G_{d}(y, z, x)=G_{d}(z, x, y)=, \cdots$, (symmetry in all three variables).

It should be observed that our notion of a G-symmetric space is the same as that of G-metric space (Definition 1.1) without the rectangular property $\left(G_{5}\right)$.

Example 1.4: Let $X=[0,1]$ equipped with a G-symmetric defined by:

$G_{d}(x, y, z)=(x-y)^{2}+(y-z)^{2}+(z-x)^{2}$ for all $x, y, z \in X$. Then, the pair $\left(X, G_{d}\right)$ is a G-symmetric space. This does not satisfy the rectangle inequality property of a G-metric space, hence it is not a G-metric space.

The analogue of axioms of Wilson [3] in G-symmetric space is as follows:

$\left(W_{3}\right)$ Given $\left\{x_{n}\right\}, x$ and $y$ in $X ; G_{d}\left(x_{n}, x, x\right) \rightarrow 0$ and $G_{d}\left(x_{n}, y, y\right) \rightarrow 0$ imply that $x=y$.

$\left(W_{4}\right)$ Given $\left\{x_{n}\right\},\left\{y_{n}\right\}$ and an $x$ in $X ; G_{d}\left(x_{n}, x, x\right) \rightarrow 0$ and $G_{d}\left(x_{n}, y_{n}, y_{n}\right) \rightarrow 0$ imply that

$G_{d}\left(y_{n}, x, x\right) \rightarrow 0$.

Definition 1.5: Let $\left(X, G_{d}\right)$ be a G-symmetric space.

(i) $\left(X, G_{d}\right)$ is $G_{d}$-complete if for every $G_{d}$-Cauchy sequence $\left\{x_{n}\right\}$, there exists $x$ in $X$ with $\lim _{n \rightarrow \infty} G_{d}\left(x_{n}, x, x\right)=0$.

(ii) $f: X \rightarrow X$ is $G_{d}$-continuous if

$$
\lim _{n \rightarrow \infty} G_{d}\left(x_{n}, x, x\right)=0 \Rightarrow \lim _{n \rightarrow \infty} G_{d}\left(f x_{n}, f x, f x\right)=0 .
$$

Definition 1.6: Let $A$ be a nonempty subset of $X . A$ is said to be $G_{d}$-bounded if and only if

$$
D=\sup \left\{G_{d}(x, y, z): x, y, z \in X\right\}<\infty .
$$

The principle of studying the fixed point of contractive maps without continuity at each point of the set was initiated by Kannan [6] in 1968. The establishment of a common fixed point for a contractive pair of commuting maps was proved by Jungck [7]. Thereafter, Sessa [8] introduced the notion of weakly commuting maps. Jungck 
[9] introduced the concept of compatible maps which is more general than the weakly commuting maps. Jungck further weakened the notion of compatibility by introducing weakly compatibility. Al-Thagafi and Shahzad [10] defined the notion of occasionally weakly compatible maps which is more general than that of weakly compatible maps. Pant [11] further introduced the concept of non-compatible maps. The importance of non-compatibility is that it permits the existence of the common fixed points for the class of Lipschitz type mapping pairs without assuming continuity of the mappings involved or completeness of the space. In 2002, Aamri and El Moutawakil [12] introduced the (E-A) property and thus generalized the concept of non-compatible maps.

This work proves the existence of a unique common fixed point for pairs of occasionally weakly compatible maps defined on a G-symmetric space satisfying some strict contractive conditions. The work generalized many known results in literature.

The following definitions are important for our study.

Definition 1.9: Two selfmaps $f$ and $g$ in a G-symmetric space $\left(X, G_{d}\right)$ are said to be weakly compatible if they commute at their points of coincidence, that is, if $f x=g x$ for some $x \in X$, then $f g x=g f x$.

Definition 1.11 [10]: Two self maps $f$ and $g$ of a set $X$ are occasionally weakly compatible if and only if there is a point $x$ in $X$ which is a coincidence point of $f$ and $g$ at which $f$ and $g$ commute.

Lemma 1.12 [13]: Let $X$ be a set, $f, g$ occasionally weakly compatible self maps of $X$. If $f$ and $g$ have a unique point of coincidence, $w:=f x=g x$, then $w$ is the unique common fixed point of $f$ and $g$.

The existence of some common fixed point results for two generalized contractive maps in a symmetric (semimetric) space satisfying certain contractive conditions were proved by Hicks and Rhoades [4] and Imdad et al. [14]. Jungck and Rhoades [13] proved the existence of common fixed points for two pairs of occasionally weakly compatible mappings defined on symmetric spaces by using a short process of obtaining the unique common fixed point of the maps. Bhatt et al. [15] proved the existence and uniqueness of a common fixed point for pairs of maps defined on symmetric spaces without using the (E-A) property and completeness, under a relaxed condition by assuming symmetry only on the set of points of coincidence. Abbas and Rhoades [16] proved the existence of a unique common fixed point for a class of operators called occasionally weakly compatible maps defined on a symmetric space satisfying a generalized contractive condition.

In this work, the existence of common fixed points for two occasionally weakly compatible maps satisfying certain contractive conditions in a G-symmetric space is proved. Our results are analogue of the result of Abbas and Rhoades [16] and an improvement of the results of Imdad et al. [14] and others in literature.

\section{Main Results}

Theorem 2.1: Let $G_{d}$ be a bounded G-symmetric for $X$. Suppose $\left(X, G_{d}\right)$ is $G_{d}$-complete and $f: X \rightarrow X$ is $G_{d}$-continuous. Then $f$ has a fixed point if and only if there exists $k \in[0,1)$ and a $G_{d}$ -continuous func- tion $g: X \rightarrow X$ which is compatible with $f$ and satisfies $g(X) \subseteq f(X)$ and

$$
G_{d}(g x, g y, g y) \leq \alpha G_{d}(f x, f y, f y) .
$$

For all $x, y \in X$. Moreover, suppose $g, f$ are occasionally weakly compatible, then $f$ and $g$ have a unique common fixed point.

Proof: Suppose $f(a)=a$ for some $a \in X$, put $g(x)=a$ for all $x \in X$. Then the conditions of the theorem are satisfied.

Conversely, suppose there exists $k$ and $g$ so that Equation (1) holds. Let $M=\operatorname{Sup}\left\{G_{d}(x, y, y): x, y \in X\right\}$. Suppose $x_{0} \in X$ is arbitrarily chosen. $x_{1}$ can be chosen such that $f x_{1}=g x_{0}$. Continuing in this process, $\left\{x_{n}\right\}$ can be chosen such that $f x_{n}=g x_{n-1}$. Using Equation (1) and the sequence $\left\{f x_{n}\right\}$,

$$
\begin{aligned}
G_{d}\left(f x_{n}, f x_{n+m}, f x_{n+m}\right) & =G_{d}\left(g x_{n-1}, g x_{n+m-1}, g x_{n+m-1}\right) \\
& \leq k G_{d}\left(f x_{n-1}, f x_{n+m-1}, f x_{n+m-1}\right) \\
& \leq k^{2} G_{d}\left(f x_{n-2}, f x_{n+m-2}, f x_{n+m-2}\right) \\
& \leq \cdots \leq k^{n} G_{d}\left(f x_{0}, f x_{m}, f x_{m}\right) \leq k^{n} M .
\end{aligned}
$$

Thus $\left\{f x_{n}\right\}$ is a $G_{d}$-Cauchy sequence and since $\left(X, G_{d}\right)$ is $G_{d}$-complete, there exists $x \in X$ with 
$\lim _{n \rightarrow \infty} G_{d}\left(f x_{n}, x, x\right)=0$. Since $g$ is $G_{d}$-continuous, it implies that $\lim _{n \rightarrow \infty} G_{d}\left(g f x_{n}, g x, g x\right)=0$. Also $f x_{n}=g x_{n-1}$ yields $\lim _{n \rightarrow \infty} G_{d}\left(g x_{n}, x, x\right)=0 . f$ is $G_{d}$-continuous implying that $\lim _{n \rightarrow \infty} G_{d}\left(f g x_{n}, f x, f x\right)=0$. The compatibility of $f$ and $g$ gives $\lim _{n \rightarrow \infty} G_{d}\left(f g x_{n}, g f x_{n}, g f x_{n}\right)=0$, that is $G_{d}(f x, g x, g x)=0$ which implies that $f x=g x$. Suppose there exists another point in $X$ saying $u$ such that $f u=g u$. Now we claim that $f x=f u$. Suppose $f x \neq f u$, then using Equation (1) gives

$$
G_{d}\left(f g x_{n}, f u, f u\right)=G_{d}\left(g g x_{n-1}, g u, g u\right) \leq k G_{d}\left(f g x_{n-1}, f u, f u\right) .
$$

Letting $n \rightarrow \infty$ yields

$$
G_{d}(f x, f u, f u) \leq k G_{d}(f x, f u, f u) .
$$

This is a contradiction since $0 \leq k<1$, hence $f x=f u$. Therefore $v=f x=g x$ is the unique point of coincidence $f$ and $g$. By Lemma (1.12), $v$ is the unique common fixed point of $f$ and $g$.

Corollary 2.2 [15]: Let $G_{d}$ be a bounded $G_{d}$-symmetric for $X$ that satisfies $\left(W_{4}\right)$. Suppose that $\left(X, G_{d}\right)$ is $G_{d}$-complete and $f: X \rightarrow X$ is $G_{d}$-continuous. Then $f$ has a fixed point if and only if there exists $k \in(0,1)$ and a $G_{d}$-continuous function $g: X \rightarrow X$ which commutes with $f$ and satisfies $g(X) \subseteq f(X)$ and

$$
G_{d}(g x, g y, g y) \leq \alpha G_{d}(f x, f y, f y),
$$

for all $x, y \in X$. Indeed, $f$ and $g$ have a unique common fixed point if Equation (2) holds.

Remark 2.3: Corollary 2.2 is an analogue of ([15], Theorem 2.1) in the setting of G-symmetric space. Theorem 2.1 is an improvement of Bhatt et al. ([15], Theorem 2.1) since occasionally weakly compatible maps are more general than commuting maps and the concept of a $G_{d}$-symmetric space extends that of a symmetric space.

Theorem 2.4: Let $\left(X, G_{d}\right)$ be a $G_{d}$-symmetric space that satisfies $\left(W_{3}\right)$. Let $f$ and $g$ be two selfmappings of $X$ such that

(i) $f$ and $g$ satisfy property (E-A),

(ii) for all $x \neq y \in X$.

Suppose

$$
\begin{aligned}
& G_{d}(g x, g y, g y)<\max {\left[G_{d}(f x, f y, f y), \frac{k}{2}\left[G_{d}(g x, f x, f x)+G_{d}(g y, f y, f y)\right],\right.} \\
&\left.\frac{k}{2}\left[G_{d}(g y, f x, f x)+G_{d}(g x, f y, f y)\right]\right]
\end{aligned}
$$

And

$$
\begin{gathered}
G_{d}(g x, g x, g y)<\max \left[G_{d}(f x, f x, f y), \frac{k}{2}\left[G_{d}(g y, g y, f y)+G_{d}(g x, g x, f x)\right],\right. \\
\left.\frac{k}{2}\left[G_{d}(g x, g x, f y)+G_{d}(g y, g y, f x)\right]\right] .
\end{gathered}
$$

$0 \leq k<1$. Suppose $f(X)$ is a $G_{d}$-closed subset of $X$ with $g(X) \subseteq f(X)$. If $f$ and $g$ are occasionally weakly compatible, then $f$ and $g$ have a unique common fixed point.

Proof: Since $f$ and $g$ satisfy property (E-A), there exists a sequence $\left\{x_{n}\right\}$ in $X$ such that $\lim _{n \rightarrow \infty} f x_{n}=$ $\lim _{n \rightarrow \infty} g x_{n}=t$ for some $t \in X$. Also $f(X)$ is closed implying that there exist some $a \in X$ such that $\lim _{n \rightarrow \infty} f x_{n}=f(a)$. This yields that $t=f(a) \in f(X)$ by $\left(W_{3}\right)$. We claim that $f(a)=g(a)$. Suppose $f(a) \neq g(a)$, then using Equation (3) we get,

$$
\begin{aligned}
G_{d}\left(g x_{n}, g a, g a\right)<\max & {\left[G_{d}\left(f x_{n}, f a, f a\right), \frac{k}{2}\left[G_{d}\left(g x_{n}, f x_{n}, f x_{n}\right)+G_{d}(g a, f a, f a)\right],\right.} \\
& \left.\frac{k}{2}\left[G_{d}\left(g a, f x_{n}, f x_{n}\right)+G_{d}\left(g x_{n}, f a, f a\right)\right]\right] .
\end{aligned}
$$

Letting $n \rightarrow \infty$ we have, 


$$
\begin{aligned}
G_{d}(f a, g a, g a)< & \max \left[G_{d}(f a, f a, f a), \frac{k}{2}\left[G_{d}(f a, f a, f a)+G_{d}(g a, f a, f a)\right],\right. \\
& \left.\frac{k}{2}\left[G_{d}(g a, f a, f a)+G_{d}(f a, f a, f a)\right]\right] \\
< & G_{d}(g a, f a, f a) .
\end{aligned}
$$

Using Equation (4) we have

$$
\begin{aligned}
G_{d}\left(g x_{n}, g x_{n}, g a\right)<\max [ & G_{d}\left(f x_{n}, f x_{n}, f a\right), \frac{k}{2}\left[G_{d}(g a, g a, f a)+G_{d}\left(g x_{n}, g x_{n}, f x_{n}\right)\right], \\
& \left.\frac{k}{2}\left[G_{d}\left(g x_{n}, g x_{n}, f a\right)+G_{d}\left(g a, g a, f x_{n}\right)\right]\right] .
\end{aligned}
$$

Letting $n \rightarrow \infty$ gives,

$$
\begin{aligned}
G_{d}(f a, f a, g a)<\max \left[G_{d}(f a, f a, f a), \frac{k}{2}\left[G_{d}(g a, g a, f a)+G_{d}(f a, f a, f a)\right],\right. \\
\left.\quad \frac{k}{2}\left[G_{d}(f a, f a, f a)+G_{d}(g a, g a, f a)\right]\right] \\
<G_{d}(g a, g a, f a)
\end{aligned}
$$

Combining Equations (5) and (6) yields,

$$
G_{d}(f a, g a, g a)<G_{d}(f a, g a, g a) .
$$

Suppose there exists $b \in X$ such that $g(b)=f(b)$. Suppose $g(b) \neq g(a)$, then using Equation (3) we have,

$$
\begin{gathered}
G_{d}\left(g x_{n}, g b, g b\right)<\max \left[G_{d}\left(f x_{n}, f b, f b\right), \frac{k}{2}\left[G_{d}\left(g x_{n}, f x_{n}, f x_{n}\right)+G_{d}(g b, f b, f b)\right],\right. \\
\left.\frac{k}{2}\left[G_{d}\left(g b, f x_{n}, f x_{n}\right)+G_{d}\left(g x_{n}, f b, f b\right)\right]\right] .
\end{gathered}
$$

Letting $n \rightarrow \infty$ yields,

$$
\begin{aligned}
& G_{d}(g a, g b, g b)< \max \left[G_{d}(f a, f b, f b), \frac{k}{2}\left[G_{d}(g a, f a, f a)+G_{d}(g b, f b, f b)\right],\right. \\
&\left.\frac{k}{2}\left[G_{d}(g b, f a, f a)+G_{d}(g a, f b, f b)\right]\right] \\
&< \max \left[G_{d}(g a, g b, g b), \frac{k}{2}\left[G_{d}(g a, g a, g a)+G_{d}(g b, g b, g b)\right],\right. \\
&\left.\frac{k}{2}\left[G_{d}(g b, g a, g a)+G_{d}(g a, g b, g b)\right]\right] \\
&< \frac{k}{2}\left[G_{d}(g b, g a, g a)+G_{d}(g a, g b, g b)\right] . \\
&(2-k) G_{d}(g a, g b, g b)<k G_{d}(g b, g a, g a), \\
& G_{d}(g a, g b, g b)<\frac{k}{2-k} G_{d}(g b, g a, g a) .
\end{aligned}
$$

Using Equation (4), we obtain 


$$
\begin{aligned}
G_{d}(g a, g a, g b)< & \max \left[G_{d}(f a, f a, f b), \frac{k}{2}\left[G_{d}(g a, g a, f a)+G_{d}(g b, g b, f b)\right],\right. \\
& \left.\frac{k}{2}\left[G_{d}(g b, g b, f a)+G_{d}(g a, g a, f b)\right]\right] \\
< & \max \left[G_{d}(g a, g a, g b), \frac{k}{2}\left[G_{d}(g b, g b, g a)+G_{d}(g a, g a, g b)\right]\right] \\
< & \frac{k}{2}\left[G_{d}(g b, g b, g a)+G_{d}(g a, g a, g b)\right] . \\
& (2-k) G_{d}(g a, g a, g b)<k G_{d}(g b, g b, g a), \\
& G_{d}(g a, g b, g b)<\frac{k}{2-k} G_{d}(g b, g b, g a) .
\end{aligned}
$$

Combining Equations (7) and (8) gives,

$$
G_{d}(g a, g b, g b)<\left(\frac{k}{2-k}\right)^{2} G_{d}(g a, g b, g b) .
$$

Since $0 \leq k<1$, we obtain $g a=g b$. Therefore $g a=f a=g b=f b$. Hence $w$ is the unique point of coincidence of $f$ and $g$. By Lemma 1.12, $w$ is the unique common fixed point of $f$ and $g$.

Corollary 2.5: Let $\left(X, G_{d}\right)$ be a $G_{d}$-symmetric space that satisfies $\left(W_{3}\right)$. Let $f$ and $g$ be two self-mappings of $X$ such that

(i) $f$ and $g$ satisfy property (E.A)

(ii) for all $x \neq y \in X$

$$
\begin{gathered}
G_{d}(g x, g y, g y)<\max \left[G_{d}(f x, f y, f y), \frac{k}{2}\left[G_{d}(g x, f x, f x)+G_{d}(g y, f y, f y)\right],\right. \\
\left.\frac{k}{2}\left[G_{d}(g y, f x, f x)+G_{d}(g x, f y, f y)\right]\right] .
\end{gathered}
$$

and

$$
\begin{aligned}
G_{d}(g y, g x, g x)<\max [ & G_{d}(f y, f x, f x), \frac{k}{2}\left[G_{d}(g y, f y, f y)+G_{d}(g x, f x, f x)\right], \\
& \frac{k}{2}\left[G_{d}(g x, f y, f y)+G_{d}(g y, f x, f x)\right] .
\end{aligned}
$$

$0 \leq k<1$. Assume $f(X)$ is $G_{d}$-closed subsets of $X$ with $g(X) \subseteq f(X)$. Suppose that $f$ and $g$ are weakly compatible, then $f$ and $g$ have a unique common fixed point.

Remarks 2.6: Theorem 2.4 is an extension of ([14], Theorems 2.1, 2.2, 2.3) to G-symmetric spaces from symmetric spaces.

The following results are analogue of ([16] Theorem 1).

First we state the following definitions given by Abbas and Rhoades [16].

Let $A \in(0, \infty], \quad R_{A}^{+}=[0, A)$. Let $F: R_{A}^{+} \rightarrow R$ satisfy

(i) $F(0)=0$ and $F(t)>0$ for each $t \in(0, A)$ and

(ii) $F$ is nondecreasing on $R_{A}^{+}$.

Define $F[0, A)=\left\{F: R_{A}^{+} \rightarrow R: F\right.$ satisfies (i) - (ii) $\}$.

Let $\psi: R_{A}^{+} \rightarrow R$ satisfy

(i) $\psi(t)<t$ for each $t \in[0, A)$ and

(ii) $\psi$ is nondecreasing.

Define $\Psi[0, A)=\left\{\psi: R_{A}^{+} \rightarrow R: \psi\right.$ satisfies (i) - (ii) above $\}$.

Theorem 2.6: Let $X$ be a set with $G_{d}$-symmetric $G_{d}$. Let $D=\operatorname{Sup}\left\{G_{d}(x, y, y): x, y \in X\right\}$. Suppose that $f, g, S$ and $T$ are self-maps of $X$ and that the pairs $\{f, S\}$ and $\{g, T\}$ are each occasionally weakly 
compatible. If for each $x, y \in X$ for which $f x \neq g y$ we have

$$
F\left(G_{d}(f x, g y, g y)\right)<\psi(F(M(x, y, y)))
$$

and

$$
F\left(G_{d}(f x, f x, g y)\right)<\psi(F(M(x, x, y))),
$$

for each $x, y \in X, \quad F \in F[0, A)$ and $\psi \in \psi[0, A)$, where $A=D$ if $D=\infty$ and $A>D$ if $D<\infty$, and

$$
M(x, y, y):=\max \left\{G_{d}(S x, T y, T y), G_{d}(S x, f x, f x), G_{d}(T y, g y, g y), G_{d}(S x, g y, g y), G_{d}(T y, f x, f x)\right\}
$$

and

$$
M(x, x, y):=\max \left\{G_{d}(S x, S x, T y), G_{d}(S x, S x, f x), G_{d}(T y, T y, g y), G_{d}(S x, S x, g y), G_{d}(T y, T y, f x)\right\} .
$$

then there is a unique point $w \in X$ such that $f w=g w=w$ and a unique point $z \in X$ such that $g z=T z=z$. Moreover, $z=w$, so that there is a unique common fixed point of $f, g, S$ and $T$.

Proof: Since the pairs $\{f, S\}$ and $\{g, T\}$ are each occasionally weakly compatible, then there exist $x, y \in X$ such that $f x=S x$ and $g y=T y$. We claim that $f x=g y$. On the contrary, suppose $f x \neq g y$ then

$$
\begin{aligned}
M(x, y, y) & :=\max \left\{G_{d}(S x, T y, T y), G_{d}(S x, f x, f x), G_{d}(T y, g y, g y), G_{d}(S x, g y, g y), G_{d}(T y, f x, f x)\right\} \\
& =\max \left\{G_{d}(f x, g y, g y), G_{d}(f x, f x, f x), G_{d}(g y, g y, g y), G_{d}(f x, g y, g y), G_{d}(g y, f x, f x)\right\} \\
& =\max \left\{G_{d}(f x, g y, g y), G_{d}(g y, f x, f x)\right\}
\end{aligned}
$$

Case (i)

If $\max \left\{G_{d}(f x, g y, g y), G_{d}(g y, f x, f x)\right\}=G_{d}(f x, g y, g y)$, then Equation (11) becomes

$$
F\left(G_{d}(f x, g y, g y)\right)<\psi(F(M(x, y, y)))=\psi\left(F\left(G_{d}(f x, g y, g y)\right)\right)<F\left(G_{d}(f x, g y, g y)\right) .
$$

Case (ii)

If $\max \left\{G_{d}(f x, g y, g y), G_{d}(g y, f x, f x)\right\}=G_{d}(g y, f x, f x)$, then Equation (11) becomes

$$
\begin{aligned}
& F\left(G_{d}(f x, g y, g y)\right)<\psi(F(M(x, y, y)))=\psi\left(F\left(G_{d}(g y, f x, f x)\right)\right)<F\left(G_{d}(g y, f x, f x)\right) . \\
M(x, x, y) & :=\max \left\{G_{d}(S x, S x, T y), G_{d}(S x, S x, f x), G_{d}(T y, T y, g y), G_{d}(S x, S x, g y), G_{d}(T y, T y, f x)\right\} \\
& =\max \left\{G_{d}(f x, f x, g y), G_{d}(f x, f x, f x), G_{d}(g y, g y, g y), G_{d}(f x, f x, g y), G_{d}(g y, g y, f x)\right\} \\
& =\max \left\{G_{d}(f x, f x, g y), G_{d}(g y, g y, f x)\right\} .
\end{aligned}
$$

Case (iii)

If $\max \left\{G_{d}(f x, f x, g y), G_{d}(g y, g y, f x)\right\}=G_{d}(f x, f x, g y)$, then Equation (12) becomes,

$$
F\left(G_{d}(f x, f x, g y)\right)<\psi(F(M(x, x, y)))=\psi\left(F\left(G_{d}(f x, f x, g y)\right)\right)<F\left(G_{d}(f x, f x, g y)\right) .
$$

Case (iv)

If $\max \left\{G_{d}(f x, f x, g y), G_{d}(g y, g y, f x)\right\}=G_{d}(g y, g y, f x)$, then Equation (13) becomes,

$$
F\left(G_{d}(f x, f x, g y)\right)<\psi(F(M(x, x, y)))=\psi\left(F\left(G_{d}(g y, g y, f x)\right)\right)<F\left(G_{d}(g y, g y, f x)\right) .
$$

Combining Equations (13) and (14) gives, $F\left(G_{d}(f x, g y, g y)\right)<F\left(G_{d}(f x, g y, g y)\right)$-a contradiction. Therefore $f x=g y$. That is, $f x=S x=g y=T y$.

Moreover, if there is another point $u$ such that $f u=S u$, then, using Equations (12) and (13) it follows that $f u=S u=g y=T y$ or $f u=f x$, and $w=f x=S x$ is a unique point of coincidence of $f$ and $S$. By Lemma $1.12, w$ is the only common fixed point of $f$ and $S$. That is $w=f w=S w$. Similarly there is a unique point $z \in X$ such that $z=g z=T z$. Suppose that $w \neq z$ then using Equation (12) we have,

$$
F\left(G_{d}(w, z, z)\right)<\psi(F(M(w, z, z)))=\psi\left(F\left(G_{d}(w, z, z)\right)\right)<F\left(G_{d}(z, w, w)\right) .
$$

Using Equation (12) we get, 


$$
F\left(G_{d}(w, w, z)\right)<\psi(F(M(w, w, z)))=\psi\left(F\left(G_{d}(w, w, z)\right)\right)<F\left(G_{d}(z, z, w)\right) .
$$

Combining Equations (15) and (16) gives,

$$
F\left(G_{d}(w, z, z)\right)<F\left(G_{d}(w, z, z)\right) .
$$

a contradiction. Therefore $w=z$ and $w$ is a common fixed point of $f, g, S$ and $T$. Following the preceding argument, it is clear that $w$ is unique.

Remarks 2.7: Theorem 2.2 is an analogue of ([16] Theorem 1$)$ in the setting of G-symmetric spaces.

Corollary 2.7: Let $X$ be a set with $G$-symmetric $G_{d}$. Let $D=\operatorname{Sup}\left\{G_{d}(x, y, y): x, y \in X\right\}$. Suppose that $f, g, S$ and $T$ are self-maps of $X$ and that the pairs $\{f, S\}$ and $\{g, T\}$ are each occasionally weakly compatible (owc). If for each $x, y \in X$ for which $f x \neq g y$ we have

$$
F\left(G_{d}(f x, g y, g y)\right) \leq \psi(F(M(x, y, y))) .
$$

and

$$
F\left(G_{d}(f x, f x, g y)\right) \leq \psi(F(M(x, x, y))) .
$$

for each $x, y \in X, F \in F[0, A)$ and $\psi \in \psi[0, F(0, A))$, where $A=D$ if $D=\infty$ and $A>D$ if $D<\infty$, and

$$
M(x, y, y):=\max \left\{k G_{d}(S x, T y, T y), k G_{d}(S x, f x, f x), k G_{d}(T y, g y, g y), \frac{\left[k G_{d}(S x, g y, g y)+k G_{d}(T y, f x, f x)\right]}{2}\right\}
$$

and

$$
M(x, x, y):=\max \left\{k G_{d}(S x, S x, T y), k G_{d}(S x, S x, f x), k G_{d}(T y, T y, g y), \frac{\left[k G_{d}(S x, S x, g y)+k G_{d}(T y, T y, f x)\right]}{2}\right\}
$$

and $0 \leq k<1$, then $f, g, S$ and $T$ have a unique common fixed point.

Proof: Since Equations (17) and (18) are special cases of Equations (11) and (12), then the proof of the corollary follows immediately from Theorem 2.6.

\section{Acknowledgements}

The authors would like to appreciate the Deanship of Scientific Research for supporting this work through their careful editing of this manuscript.

\section{References}

[1] Eke, K.S. and Olaleru, J.O. (2013) Some Fixed Point Results on Ordered G-Partial Metric Spaces. ICASTOR Journal of Mathematical Sciences, 7, 65-78.

[2] Cartan, E. (1929) Sur la Determination d'un Systeme Orthogonal Complet dans un Espace de Riemman Symetrique Clos. Rendiconti del Circolo Matematico di Palermo, 53, 217-252. http://dx.doi.org/10.1007/BF03024106

[3] Wilson, W.A. (1931) On Semi-Metric Spaces. American Journal of Mathematics, 53, 361-373. http://dx.doi.org/10.2307/2370790

[4] Hicks, T.I. and Rhoades, B.E. (1999) Fixed Point Theory in Symmetric Spaces with Applications to Probabilistic Spaces. Nonlinear Analysis, 36, 331-344. http://dx.doi.org/10.1016/S0362-546X(98)00002-9

[5] Mustafa, Z. and Sims, B. (2006) A New Approach to Generalized Metric Spaces. Journal of Nonlinear and Convex Analysis, 7, 289-297.

[6] Kannan, R. (1968) Some Results on Fixed Points. Bulletin Calcutta Mathematical Society, 10, 71-76.

[7] Jungck, G. (1976) Commuting Mappings and Fixed Points. American Mathematics Monthly, 73, 261-263. http://dx.doi.org/10.2307/2318216

[8] Sessa, S. (1982) On a Weak Commutativity Condition of Mappings in Fixed Point Considerations. Publications of Institutional Mathematics, 32, 149-153. 
[9] Jungck, G. (1986) Compatible Mappings and Common Fixed Points. International Journal of Mathematics and Mathematical Science, 9, 771-779. http://dx.doi.org/10.1155/S0161171286000935

[10] Al-Thagafi, M.A. and Shahzad, N. (2008) Generalised I-Nonexpansive Selfmaps and Invariant Approximations. Acta Mathematica Sinica, 24, 867-876. http://dx.doi.org/10.1007/s10114-007-5598-X

[11] Pant, R.P. (1998) Common Fixed Points of Four Maps. Bulletin Calcutta Mathematical Society, 90, 281-286.

[12] Aamri, M. and El Moutawakil, D. (2002) Some New Common Fixed Point Theorems under Strict Contractive Conditions. Journal Mathematical Analysis and Applications, 270, 181-188. http://dx.doi.org/10.1016/S0022-247X(02)00059-8

[13] Jungck, G. and Rhoades, B.E. (2006) Fixed Point Theorems for Occasionally Weakly Compatible Mappings. Fixed Point Theory, 7, 287-296.

[14] Imdad, M., Ali, J. and Khan, L. (2006) Coincidence and Fixed Points in Symmetric Spaces under Strict Contractions. Journal of Mathematical Analysis and Applications, 320, 352-360. http://dx.doi.org/10.1016/j.jmaa.2005.07.004

[15] Bhatt, A., Chandra, H. and Sahu, D.R. (2010) Common Fixed Point Theorems for Occasionally Weakly Compatible Mappings under Relaxed Conditions. Nonlinear Analysis, 73, 176-182. http://dx.doi.org/10.1016/j.na.2010.03.011

[16] Abbas, M. and Rhoades, B.E. (2008) Common Fixed Point Theorems for Occasionally Weakly Compatible Mappings Satisfying a Generalized Contractive Condition. Mathematical Communication, 13, 295-301. 